\title{
MANIFESTATION OF PROTON STRUCTURE IN RIDGE-LIKE CORRELATIONS IN HIGH-ENERGY PROTON-PROTON COLLISIONS
}

\author{
P. Kubiczek ${ }^{\mathrm{a}}$ and S.D. Głazek ${ }^{\mathrm{b}}$ \\ ${ }^{a}$ Faculty of Physics, Astronomy and Applied Computer Science, Jagiellonian University, \\ Lojasiewicza 11, 30-348 Kraków, Poland \\ ${ }^{\mathrm{b}}$ Institute of Theoretical Physics, Faculty of Physics, University of Warsaw, Pasteura 5, 02-093 Warszawa, Poland \\ E-mail: patryk.kubiczek@student.uj.edu.pl
}

Received 21 May 2015; revised 22 June 2015; accepted 29 September 2015

\begin{abstract}
Ridge-like correlations in high-energy proton-proton collisions reported by the CMS collaboration suggest a collective flow that resembles the one in heavy-ion collisions. If the hydrodynamic description is valid, then the effect results from the initial anisotropy of the colliding matter which depends on the structure of protons. Following recent theoretical developments, we propose several phenomenological models of the proton structure and calculate the anisotropy coefficients using the Monte Carlo Glauber model. Our estimates suggest that the event multiplicity dependence allows one to discriminate between different proton models.
\end{abstract}

Keywords: high-energy proton-proton collisions, two-particle correlations, collective flow, proton structure, renormalization group

PACS: 13.85.-t, 25.75.Gz, 25.75.Ld

\section{Introduction}

The analysis of two-particle angular correlations in $p p$ collisions at $\sqrt{s}=7 \mathrm{TeV}$ revealed an unexpected near side $(\Delta \phi=0)$ correlation in the azimuthal angle of produced particles ('ridge effect') [1]. There is no obvious reason why such a long-range pseudorapidity correlation should occur [2, 3]. The ridge effect has been previously observed in relativistic heavy-ion collisions and it was explained by a collective anisotropic flow of a hot and dense medium (hadronic gas or quark gluon plasma) created at the collision.

It is possible that the hydrodynamic explanation applies to high-multiplicity $p p$ events which are suficiently energetic for the collision products to thermalize [4]. In this paper we assume validity of such a scenario and postulate that the proton internal structure can manifest itself in the ridge effect. During the hydrodynamic expansion, the spatial anisotropies of the collision area are transformed into an anisotropic flow of produced particles. Thus, the spatial anisotropies due to the proton structure should be reflected in the measurable collective flow coefficients.

Actually, in the literature there exist estimates of the possible elliptic flow in $p p$ collisions [5-13]. We basically follow the approach of [5] in order to make a link between the proton models considered by us and the experimental data. Within the framework of the Glauber model [14], we calculate the initial spatial anisotropies for each model: eccentricity, denoted by $\epsilon_{2}$, and triangularity, denoted by $\epsilon_{3}$, in proton-proton collision taking into account event-by-event fluctuations in the proton density profile.

The considered phenomenological proton densities are inspired by the quark-diquark [17] and RGPEP (renormalization group procedure for effective particles) [18] models of the proton. We find that it is possible to distinguish highly eccentric (rod-like) and highly triangular (triangle-like) fixed proton densities by looking at distributions of $\epsilon_{2}$ and $\epsilon_{3}$ and the multiplicity dependence of their RMS values. We also calculate these quantities for the fluctuating anisotropic proton configurations (Gaussian-fluctuating model).

We do not explicitly present the results for the anisotropic flow coefficients $v_{n}$ because we limit our discussion to the comparison of predictions of different models for the initial states in hydrodynamic evolution, and the prediction of the exact shapes of the resulting final flow anisotropies is beyond the scope of this work. Our calculations concern the initial spatial anisotropies, though we emphasize that they should correspond to the flow coefficients measurable in 
multi-particle correlations in a fashion that is unlikely to be far from linear [15, 16].

\section{Glauber model for $p p$ collisions}

The optical limit of the Glauber model treats the collision of two composite particles as a superposition of independent binary collisions between their constituents. In the case of $p p$ collisions, we assume that the constituents are partons of one type, and their smoothed distribution is given by a proton density profile. We fix the partonic cross section $\sigma_{\mathrm{gg}}=4.3 \mathrm{mb}$ [15] and adjust the number of partons in proton $N_{\mathrm{g}}$ so that the experimental inelastic $p p$ cross section $\sigma_{\mathrm{pp}} \approx 60 \mathrm{mb}$ [19] is reproduced.

The geometrical quantities calculated within the Glauber formalism correspond to the impact plane of the collision $(x y)$ such that any $z$-dependence is integrated out. The density of binary collisions is given by the expression:

$$
\begin{aligned}
& n_{\text {coll }}\left(x, y ; b, \Sigma_{A}, \Sigma_{B}\right)= \\
& \sigma_{\mathrm{gg}} \int_{-\infty}^{\infty} \mathrm{d} z \rho\left(x-\frac{b}{2}, y, z ; \Sigma_{A}\right) \int_{-\infty}^{\infty} \mathrm{d} z^{\prime} \rho\left(x+\frac{b}{2}, y, z^{\prime} ; \Sigma_{B}\right),
\end{aligned}
$$

where $\rho(x, y, z ; \Sigma)$ is a proton density profile, $b$ is an impact parameter, and by $\Sigma$ we mean a set of fluctuating parameters describing the proton internal structure and orientation in space (for fixed configuration models $\Sigma$ dependence will correspond only to possible rotations in space) [14.

For each event characterized by $b, \Sigma_{A}$ and $\Sigma_{B}$ we calculate eccentricity $\epsilon_{2}$ and triangularity $\epsilon_{3}$. We choose the following definition of spatial anisotropy [20]:

$$
\epsilon_{\mathrm{n}}=\frac{\sqrt{\left\{s^{n} \cos (n \varphi)\right\}^{2}+\left\{s^{n} \sin (n \varphi)\right\}^{2}}}{\left\{s^{n}\right\}},
$$

where $\phi$ is an azimuthal angle in the $x y$ plane and $s^{2}=x^{2}+y^{2}$. The curly brackets $\{\cdots\}$ stand for the average taken with respect to the impact plane binary collision density $n_{\text {coll }}\left(x, y ; b, \Sigma_{A}, \Sigma_{B}\right)$ :

$$
\{f(x, y)\}=\frac{\int \mathrm{d} x \mathrm{~d} y f(x, y) n_{\text {coll }}\left(x, y ; b, \Sigma_{A}, \Sigma_{B}\right)}{\int \mathrm{d} x \mathrm{~d} y n_{\text {coll }}\left(x, y ; b, \Sigma_{A}, \Sigma_{B}\right)} .
$$

The differential cross section (probability) of a given event depends only on the integrated number of binary collisions $N_{\text {coll }}\left(b, \Sigma_{A}, \Sigma_{B}\right)=\int \mathrm{d} x \mathrm{~d} y n_{\text {coll }}\left(x, y ; b, \Sigma_{A}, \Sigma_{B}\right)$ :

$$
\sigma\left(b, \Sigma_{A}, \Sigma_{B}\right)=\left[1-\frac{N_{\text {coll }}\left(b, \Sigma_{A}, \Sigma_{B}\right)}{N_{\mathrm{g}}^{2}}\right]^{N_{\mathrm{g}}^{2}} .
$$

The expectation value of the quantity $Q$ is calculated in the following way:

$$
\begin{aligned}
& \langle Q\rangle=\frac{1}{\sigma_{\mathrm{pp}}} \int_{0}^{\infty} 2 \pi b \mathrm{~d} b \int P\left(\Sigma_{A}\right) \mathrm{d} \Sigma_{A} \\
& \int P\left(\sum_{B}\right) \mathrm{d} \Sigma_{B} \sigma\left(b, \Sigma_{A}, \Sigma_{B}\right) Q\left(b, \Sigma_{A}, \Sigma_{B}\right),
\end{aligned}
$$

where $\sigma_{\mathrm{pp}}=\int_{0}^{\infty} 2 \pi b \mathrm{~d} b \int P\left(\Sigma_{A}\right) \mathrm{d} \Sigma_{A} \int P\left(\Sigma_{B}\right) \mathrm{d} \Sigma_{B} \sigma\left(b, \Sigma_{A}, \Sigma_{B}\right)$ and $P(\Sigma)$ is the probability density of proton configuration $\Sigma$.

We calculate the charged hadron multiplicity $N$ for each event assuming its linear scaling with $N_{\text {coll: }}$ :

$$
N=\alpha N_{\text {coll }}
$$

where $\alpha$ is determined by the demand of reproducing the experimental minimum bias charged hadron multiplicity $\langle N\rangle=30$ [21].

There is another approach to estimating the multiplicities and geometrical quantities within the Glauber formalism, namely, the wounded nucleon model [22]. Within this model, all the averages denoted by $\{\ldots\}$ are taken with respect to the local density of participating constituents instead of the density of binary collisions. However, in high energy hadronic collisions, multiple gluonic interactions are expected to occur, so we think it is more natural to use here the density of binary partonic collisions.

\section{Models of proton internal structure}

The RGPEP [18] suggests that a proton can be described in terms of three effective quarks and a gluon body interacting via a harmonic potential. For every considered special case of this picture we model the proton as a sum of isotropic 3D Gaussian densities $\rho_{\mathrm{q}}$ corresponding to effective quarks and anisotropic Gaussians $\rho_{\mathrm{g}}$ representing the gluonic flux tubes connecting the quarks:

$$
\begin{aligned}
& \rho_{\mathrm{q}}\left(\mathbf{r} ; r_{\mathrm{q}}\right)=\frac{1}{(2 \pi)^{3 / 2} r_{\mathrm{q}}^{3}} \mathrm{e}^{-\frac{r^{2}}{2 r_{\mathrm{q}}^{2}}}, \\
& \rho_{\mathrm{g}}\left(\mathbf{r} ; r_{\mathrm{s}}, r_{\mathrm{l}}\right)=\frac{1}{(2 \pi)^{3 / 2} r_{\mathrm{s}}^{2} r_{\mathrm{l}}} \mathrm{e}^{-\frac{x^{2}+y^{2}}{2 r_{\mathrm{s}}^{2}}-\frac{z^{2}}{2 r^{2}}} .
\end{aligned}
$$

The effective quarks and the gluon body are clusters of partons, as in the two stage and cluster models [23-25] used to explain the shape of deep inelastic scattering structure functions.

\subsection{Fixed configuration models: I and $Y$}

According to [17], the ridge effect could be the consequence of large eccentricities $\epsilon_{2}$ in high-multiplicity 
collisions between aligned quark-diquark states of protons which constitute around $20 \%$ of all possible states.

We model this state, labelling it with the symbol I, as a superposition of two effective quark bodies, with quark and diquark parton densities generically described in terms of simple functions, and two gluonic tubes connecting the quark bodies (Fig. 11). Such greatly simplified density model is sufficient for tracing the effects of a symmetric rod-like shape of proton in comparison with other conceivable shapes (see below), leaving the more subtle effects of the asymmetry between quark and diquark for further analysis elsewhere. Thus, we use here

$$
\begin{aligned}
& \rho_{\mathrm{I}}(\mathbf{r})=N_{\mathrm{g}} \frac{1-\kappa}{2}\left[\rho_{\mathrm{q}}\left(x, y, z-\frac{d}{2} ; r_{\mathrm{q}}\right)\right. \\
& \left.+\rho_{\mathrm{q}}\left(x, y, z+\frac{d}{2} ; r_{\mathrm{q}}\right)\right]+N_{\mathrm{g}} \frac{\kappa}{2}\left[\rho_{\mathrm{g}}\left(x, y, z-\frac{d}{4} ; r_{\mathrm{q}}, \frac{d}{4}\right)\right. \\
& \left.+\rho_{\mathrm{g}}\left(x, y, z+\frac{d}{4} ; r_{\mathrm{q}}, \frac{d}{4}\right)\right] .
\end{aligned}
$$

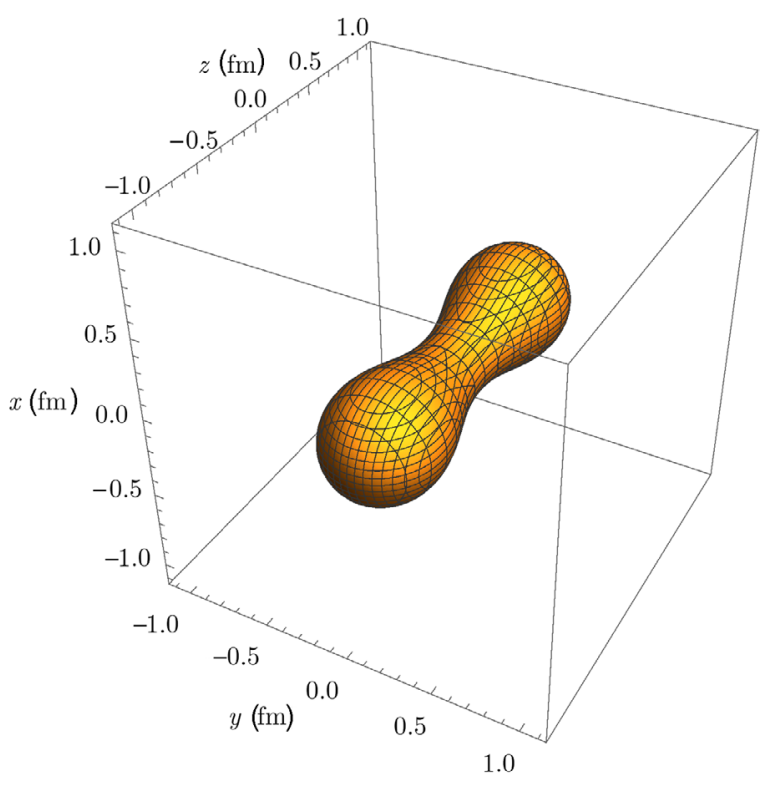

Fig. 1. Constant proton density surface for model I (quark-diquark).

The free parameters of the model are the effective quark radius $r_{q}$, the length of quark-diquark tube $d$, and the percentage of gluon body content $\kappa$. Assuming that only effective quark bodies carry net charge $(+4 / 3 e$ homogenously distributed in the diquark and $-1 / 3 e$ in the quark), we choose $r_{\mathrm{q}}=0.25 \mathrm{fm}$ and $d=1.5 \mathrm{fm}$, which reproduce the charge rms radius of proton $(\approx 0.9 \mathrm{fm})$. In this work, we always choose $\kappa=0.5$.
For comparison, we also consider a model that we label by the symbol $Y$ in which the proton has a highly triangular shape (Fig. 2). The parton density in this case is assumed in the form

$$
\begin{aligned}
& \rho_{\mathrm{Y}}(\mathbf{r})=N_{\mathrm{g}} \frac{1-\kappa}{3} \sum_{k=1}^{3} \rho_{\mathrm{q}}\left(\mathbf{r}-\mathbf{r}_{k} ; r_{\mathrm{q}}\right) \\
& +N_{\mathrm{g}} \frac{\kappa}{3} \sum_{k=1}^{3} \rho_{\mathrm{g}}\left(\mathrm{R}^{-1}\left[k \frac{2 \pi}{3}\right]\left(\mathbf{r}-\frac{\mathbf{r}_{k}}{2}\right) ; r_{\mathrm{q}}, \frac{r_{k}}{2}\right),
\end{aligned}
$$

where $\quad \mathbf{r}_{1}=\left(0,-\frac{\sqrt{3}}{4} d,-\frac{d}{4}\right), \quad \mathbf{r}_{2}=\left(0,-\frac{\sqrt{3}}{4} d,-\frac{d}{4}\right), \quad \mathbf{r}_{3}=\left(0,0, \frac{d}{2}\right)$ and $\mathrm{R}[\vartheta]$ is a rotation matrix in the $y z$ plane. In this model, the effective quarks are located in the vertices of an equilateral triangle and the gluon tubes connect them with the center of mass of the system. Again we choose $r_{\mathrm{q}}=0.25 \mathrm{fm}$ and $d=1.5 \mathrm{fm}$.

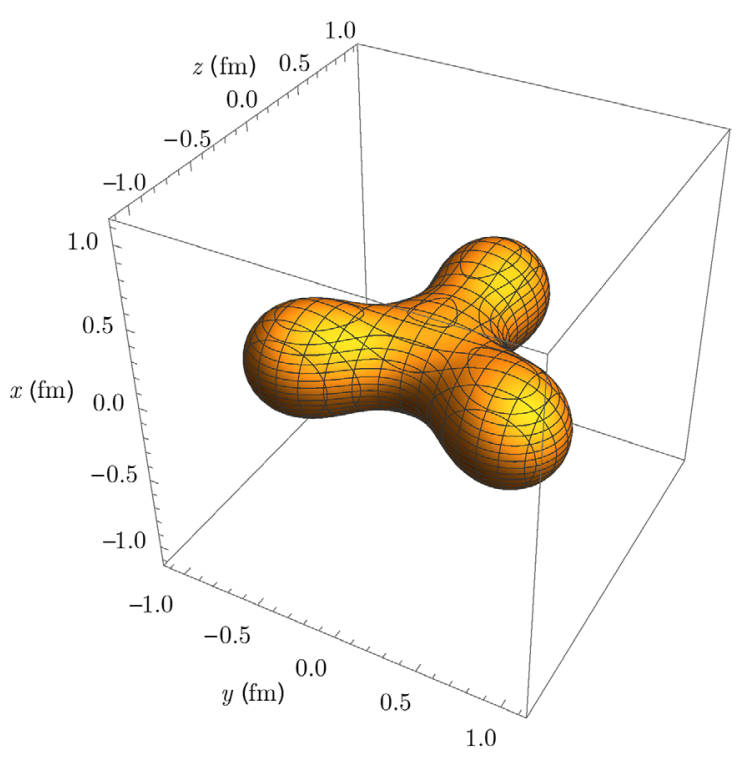

Fig. 2. Constant proton density surface for model Y.

The densities of types I and Y are fixed proton structure models in a sense that the only parameters that fluctuate event-by-event are the angles describing proton orientation relative to the direction of its velocity (not explicitly introduced above).

\subsection{Gaussian-fluctuating configuration model}

We also consider a model with a fluctuating proton configuration, labelled G-f, in which the relative positions of effective quarks differ event-by-event. By generalizing the previous formulas for proton densities, we introduce the Gaussian-fluctuating proton density 


$$
\begin{aligned}
& \rho_{\mathrm{G}-\mathrm{f}}\left(\mathbf{r} ; \mathbf{r}_{1}, \mathbf{r}_{2}, \mathbf{r}_{3}\right)=N_{\mathrm{g}} \frac{1-\kappa}{3} \sum_{k=1}^{3} \rho_{\mathrm{q}}\left(\mathbf{r}-r_{k} ; r_{\mathrm{q}}\right) \\
& +N_{\mathrm{g}} \frac{\kappa}{3} \sum_{k=1}^{3} \rho_{\mathrm{g}}\left(\mathrm{R}^{-1}\left[\theta_{k}, \phi_{k}\right]\left(\mathbf{r}-\frac{r_{k}}{2}\right) ; r_{\mathrm{q}}, \frac{r_{k}}{2}\right) .
\end{aligned}
$$

In this expression, $\mathrm{R}[\theta, \phi]$ transforms the vector $(0$, $0,1)$ into $(\cos \phi \sin \theta, \sin \phi \sin \theta, \cos \theta)$ and $\mathbf{r}_{k}=r_{k}$ $\left(\cos \phi_{k} \sin \theta_{k} \sin \phi_{k} \sin \theta_{k}, \cos \theta_{k}\right)$ is the position vector of $k$ th effective quark.

Following the harmonic oscillator phenomenology, we assume that the probability density of finding a proton whose quarks are in positions $\mathbf{r}_{1}, \mathbf{r}_{2}, \mathbf{r}_{3}$ is Gaussian with an additional constraint of in the center-ofmass frame $\left(\mathbf{r}_{1}+\mathbf{r}_{2}+\mathbf{r}_{3}=0\right.$, obtained by recentering of the quarks):

$$
P\left(\mathbf{r}_{1}, \mathbf{r}_{2}, \mathbf{r}_{3}\right)=\frac{1}{\left[(2 \pi)^{3 / 2} R_{\mathrm{P}}^{3}\right]^{3}} \mathrm{e}^{-\left(r_{1}^{2}+r_{2}^{2}+r_{3}^{2}\right) / 2 R_{\mathrm{P}}^{2}} .
$$

The free parameters in the G-f model are $\kappa, r_{\mathrm{q}}$ and $R_{\mathrm{p}}$. Again we set $r_{\mathrm{q}}=0.25 \mathrm{fm}$, which implies the value $R_{\mathrm{p}}=0.43 \mathrm{fm}$ if one wants to obtain the experimental rms proton charge radius.

\section{Results}

We present the event distribution of eccentricities $\epsilon_{2}$ and triangularities $\epsilon_{3}$ for three classes of $p p$ collision models: II, YY and G-f in which colliding protons are described by I, Y or G-f model, respectively (Fig. 3, 4).

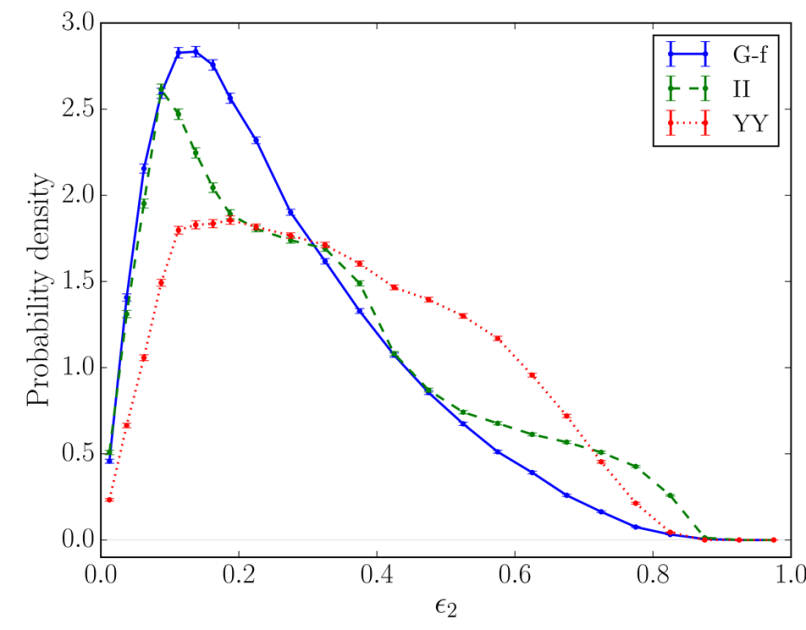

Fig. 3. Distribution of event eccentricities for II, YY and Gaussian-fluctuating type (G-f G-f) collisions. The continuous line is reconstructed from the values in particular eccentricity bins and represents the normalized probability density of the occurence of an event with certain eccentricity. Errors (square roots of variances) follow from basic statistics assuming independent events (applies to all other figures).

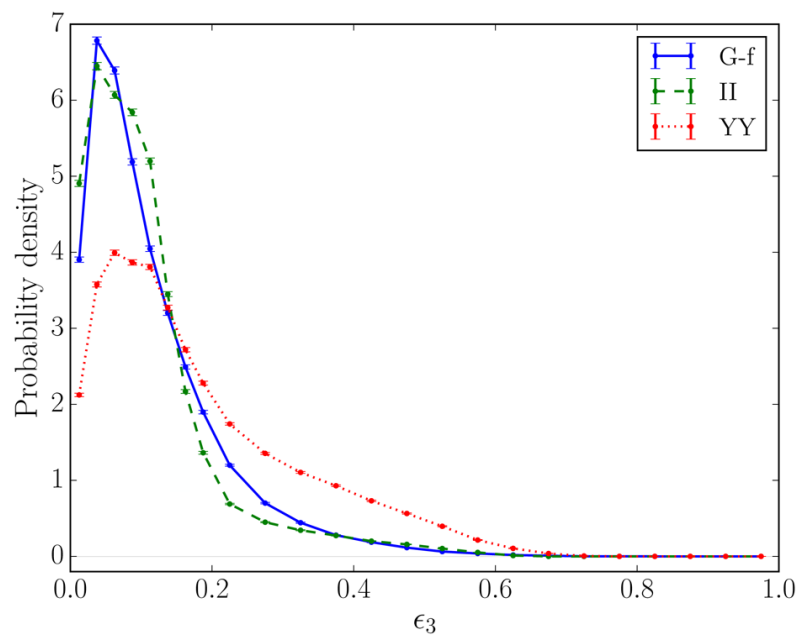

Fig. 4. Distribution of event triangularities for II, YY and Gaussian-fluctuating type (G-f G-f) collisions.

We also present the RMS values $\sqrt{\left\langle\epsilon_{2}^{2}\right\rangle}$ and $\sqrt{\left\langle\epsilon_{3}^{2}\right\rangle}$ in certain multiplicity bins (Fig. 5, 6) and the distribution of event multiplicities $N$ (Fig. Đ). The reason for presenting RMS and not mean values of anisotropies is because the former correspond hydrodynamically to the actual anisotropic flow coefficients extracted from two particle correlations (we base this approach on [12]). The minimum bias (averaged over all events)

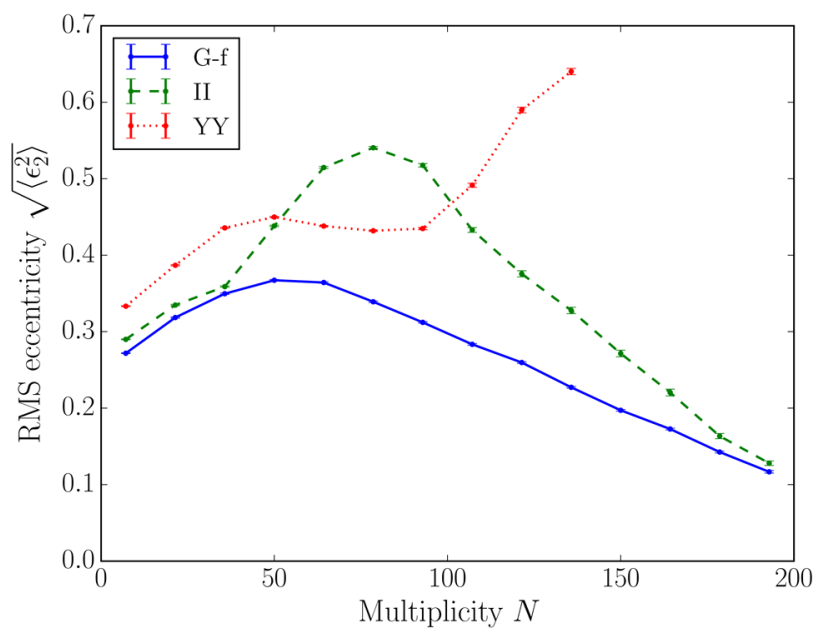

Fig. 5. RMS eccentricities in collision multiplicity bins for II, YY and Gaussian-fluctuating type (G-f G-f) collisions.

$\sqrt{\left\langle\epsilon_{2}^{2}\right\rangle_{\mathrm{MB}}}$ is $0.37,0.40$ and 0.31 for II, YY and G-f model, respectively, while $\sqrt{\left\langle\epsilon_{3}^{2}\right\rangle_{\mathrm{MB}}}=0.14,0.23,0.15$, correspondingly (these quantities can be calculated using probability densities from Figs. 3 and 4 .

All the calculations are performed by means of the Monte Carlo algorithm. 300000 events are 


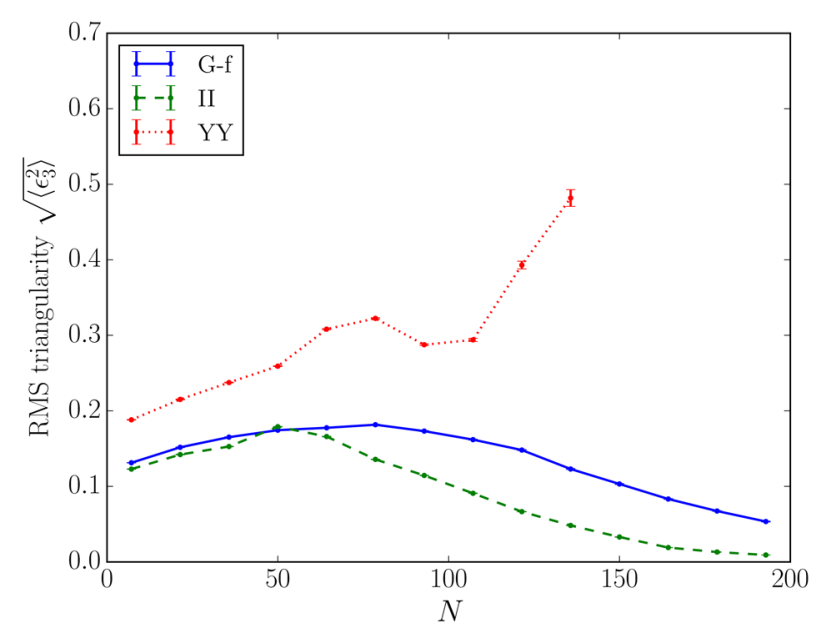

Fig. 6. RMS triangularities in collision multiplicity bins for II, YY and Gaussian-fluctuating type (G-f G-f) collisions.

generated for each class of collision. The statistical weight of an event is given by Eq. (4) and the mean values are calculated according to Eq. (5). In the case of models I and Y, we average only over the impact parameter $b$ and over all the possible space orientations of protons during the collision. In the case of G-f model, the additional averaging over the positions of effective quarks takes place. The event multiplicity $N$ is estimated by the use of Eq. (6).

We set $N_{\mathrm{g}}$ in such a way that the experimental $p p$ inelastic cross section of $60 \mathrm{mb}$ is reproduced. This is accomplished for $N_{\mathrm{g}}$ in a range of 7-10. Similarly, the parameter $\alpha$ from Eq. (6) is found to vary between 5 and 11.

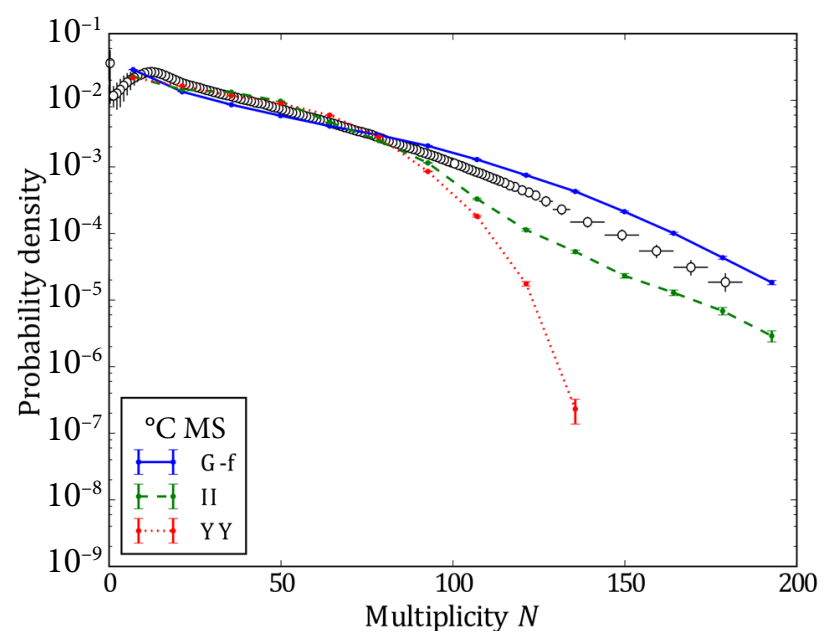

Fig. 7. Event multiplicity distributions for II, YY and Gaussian-fluctuating type collisions compared to the experimental charged hadron distribution in $\sqrt{s}=7 \mathrm{TeV} p p$ collisions [21] for the pseudorapidity range $|\eta|<2.4$.

\section{Conclusions}

Our calculations predict quite high values of eccentricities and triangularities in $p p$ collisions. Assuming the hydrodynamic limit in which the scaling between the initial spatial and finite momentum anisotropy is linear and taking $v_{2} / \epsilon_{2} \sim 0.3$ [15], we expect the minimum bias $v_{2} \sim 0.11,0.12,0.09$ for the quark-diquark, triangular and Gaussian-fluctuating model. Bożek extracts from the ridge effect $v_{2}$ in a range of 0.04-0.08 [⿰亻也 ] which can potentially be in agreement with our results if viscous effects reducing the scaling factor are taken into account.

Our models predict that one should be able to distinguish quark-diquark (I), triangular (Y) and Gaussianfluctuating (G-f) proton configurations by extracting the collective flow coefficients $v_{2}$ and $v_{3}$ from multiparticle correlations. The actual shapes of the collective flow dependence on multiplicity will differ from Figs. 5 and 6 , though it is reasonable to assume that to the first approximation the scaling of $v_{\mathrm{n}}$ with $\epsilon_{\mathrm{n}}$ is linear. In general, the anisotropies in collisions of triangular protons, denoted as YY, are much higher than in those of II or G-f G-f. This was expected because irrespective of the orientation of Y protons the collision area is always anisotropic. We find that the values of anisotropy coefficients in YY collisions increase strongly in high multiplicity bins $N$. Collisions of quark-diquark protons (II) are characterised by relatively large eccentricities $\epsilon_{2}$ (though not as large as in YY collisions) and small triangularities $\epsilon_{3}$. The distributions of anisotropies and the $N$ dependence of their mean values are the most smooth for the G-f G-f collisions, which results from the existence of additional degrees of freedom.

We observe that the fraction of high-multiplicity events in the fixed configuration models is smaller than in the Gaussian-fluctuating model. Our comparison with the experimental data (Fig. Ø) indicates that a more realistic model of $p p$ collision should include not just one type, but all II, YY, G-f G-f (and IY, IG-f, YG-f) types of collision configurations with certain probabilities. However, the G-f model alone can approximately describe the experimental results for multiplicity distributions.

Avsar et al. [12] using a DIPSY Monte Carlo generator predict values of eccentricity which are similar to our result for triangular proton configurations. The distributions of eccentricities within the 'hot spot' model [6] seem to be centered more towards higher values of $\epsilon_{2}$ than in our result. The probable reason is that the hot spot' model lacks any constraint on the relative positions of quarks. It is hard to make any direct comparison with the other mentioned calculations, however, all of them [5-13] predict measurable anisotropies in $p p$ collisions. 


\section{Summary}

In view of the great interest in understanding the proton structure, it is pointed out that even simple model ideas concerning distribution of quarks and gluons in protons lead to the non-trivial multiplicity dependence of initial anisotropies in $p p$ collisions. We illustrate this statement with the results obtained for phenomenological models intuitively inspired by RGPEP in QCD and similar ideas. Expecting new data on high-energy $p p$ collisions from the $\sqrt{s}=14 \mathrm{TeV}$ runs of the Large Hadron Collider, we believe that detailed studies of the role of proton structure models in description of such collisions may shed new light on the internal structure of protons.

\section{References}

[1] V. Khachatryan et al. [CMS Collaboration], Observation of long-range, near-side angular correlations in proton-proton collisions at the LHC, J. High Energy Phys. 1009, 091 (2010), http:// dx.doi.org/10.1007/JHEP09(2010)091

[2] H. Białkowska, The ridge effect from $p-p$ to $P b$ $\mathrm{Pb}$ (and back), Acta Phys. Pol. B 43, 705 (2012), http://dx.doi.org/10.5506/APhysPolB.43.705

[3] W. Li, Observation of a "Ridge" correlation structure in high multiplicity proton-proton collisions: a brief review, Mod. Phys. Lett. A 27, 1230018 (2012), http://dx.doi.org/10.1142/S0217732312300182

[4] P. Bożek, Elliptic flow in proton-proton collisions at $\sqrt{s}=7 \mathrm{TeV}$, Eur. Phys. J. C 71, 1530 (2011), http:// dx.doi.org/10.1140/epjc/s10052-010-1530-0

[5] D. d'Enterria, G.Kh. Eyyubova, V.L. Korotkikh, I.P. Lokhtin, S.V. Petrushanko, L.I. Sarycheva, and A.M. Snigirev, Estimates of hadron azimuthal anisotropy from multiparton interactions in protonproton collisions at $\sqrt{s}=14 \mathrm{TeV}$, Eur. Phys. J. C 66, 173 (2010), http://dx.doi.org/10.1140/epjc/s10052 009-1232-7

[6] J. Casalderrey-Solana and U.A. Wiedemann, Eccentricity fluctuations make flow measurable in high multiplicity $p-p$ collisions, Phys. Rev. Lett. 104, 102301 (2010), http://dx.doi.org/10.1103/ PhysRevLett.104.102301

[7] S.K. Prasad, V. Roy, S. Chattopadhyay, and A.K. Chaudhuri, Elliptic flow $\left(v_{2}\right)$ in $p p$ collisions at energies available at the CERN Large Hadron Collider: a hydrodynamical approach, Phys. Rev. C 82, 024909 (2010), http://dx.doi.org/10.1103/ PhysRevC.82.024909

[8] P. Bożek, Observation of the collective flow in proton-proton collisions, Acta Phys. Pol. B 41, 837 (2010), http://arxiv.org/abs/0911.2392

[9] G. Ortona, G.S. Denicol, P. Mota, and T. Kodama, Elliptic flow in high multiplicity proton-proton collisions at $\sqrt{s}=14 \mathrm{TeV}$ as a signature of deconfinement and quantum energy density fluctuations,
arXiv:0911.5158 [hep-ph] (2009), http://arxiv.org/ abs/0911.5158

[10]T. Pierog, S. Porteboeuf, I. Karpenko, and K. Werner, Collective flow in (anti)proton-proton collision at Tevatron and LHC, arXiv:1005.4526 [hep-ph] (2010), http://arxiv.org/abs/1005.4526

[11]I. Bautista, L. Cunqueiro, J. Dias de Deus, and C. Pajares, Particle production azimuthal asymmetries in a clustering of color sources model, I. Phys. G 37, 015103 (2010), http://dx.doi.org/10.1088/0954$3899 / 37 / 1 / 015103$

[12]E. Avsar, Ch. Flensburg, Y. Hatta, J.-Y. Ollitrault, and T. Ueda, Eccentricity and elliptic flow in proton-proton collisions from parton evolution, Phys. Lett. B 702, 394 (2011), http://dx.doi.org/10.1016/j. physletb.2011.07.031

[13]W.-T. Deng, Z. Xu, and C. Greiner, Elliptic and triangular flow and their correlation in ultrarelativistic high multiplicity proton-proton collisions at $14 \mathrm{TeV}$, Phys. Lett. B 711, 301 (2012), http://dx.doi. org/10.1016/j.physletb.2012.04.010

[14] M.L. Miller, K. Reygers, S.J. Sanders, and P. Steinberg, Glauber modeling in high energy nuclear collisions, Ann. Rev. Nucl. Part. Sci. 57, 205 (2007), http:// dx.doi.org/10.1146/annurev.nucl.57.090506.123020

[15]H.J. Drescher, A. Dumitru, C. Gombeaud, and J.Y. Ollitrault, the centrality dependence of elliptic flow, The hydrodynamic limit, and the viscosity of hot QCD, Phys. Rev. C 76, 024905 (2007), http:// dx.doi.org/10.1103/PhysRevC.76.024905

[16] B. Alver and G. Roland, Collision geometry fluctuations and triangular flow in heavy-ion collisions, Phys. Rev. C 81, 054905 (2010), http:// dx.doi.org/10.1103/PhysRevC.81.054905

[17]J.D. Bjorken, S.J. Brodsky, and A.S. Goldhaber, Possible multiparticle ridge-like correlations in very high multiplicity proton-proton collisions, Phys. Lett. B 726, 344 (2013), http://dx.doi.org/10.1016/j. physletb.2013.08.066

[18] S.D. Głazek, Hypothesis of quark binding by condensation of gluons in hadrons, Few-Body Syst. 52, 367 (2012), http://dx.doi.org/10.1007/s00601-011-0282-1

[19] S. Chatrchyan et al. [CMS Collaboration], Measurement of the inelastic proton-proton cross section at $\sqrt{s}=7 \mathrm{TeV}$, Phys. Lett. B 722, 5 (2013), http:// dx.doi.org/10.1016/j.physletb.2013.03.024

[20]J.-P. Blaizot, W. Broniowski, and J.-Y. Ollitrault, Correlations in the Monte Carlo Glauber model, Phys. Rev. C 90, 034906 (2014), http://dx.doi. org/10.1103/PhysRevC.90.034906

[21] V. Khachatryan et al. [CMS Collaboration], Charged particle multiplicities in $p p$ interactions at $\sqrt{s}=0.9$, 2.36 and $7 \mathrm{TeV}$, J. High Energy Phys. 1101, 079 (2011), http://dx.doi.org/10.1007/JHEP01(2011)079

[22] A. Białas, M. Błeszyński, and W. Czyż, Multiplicity distributions in nucleus-nucleus collisions at high energies, Nucl. Phys. B 111, 461 (1976), http:// dx.doi.org/10.1016/0550-3213(76)90329-1 
[23]G. Altarelli, N. Cabibbo, L. Maiani, and R. Petronzio, The nucleon as a bound state of three quarks and deep inelastic phenomena, Nucl. Phys. B 69, 531 (1974), http://dx.doi.org/10.1016/0550-3213(74)90452-0

[24]L. Van Hove and S. Pokorski, High-energy hadron-hadron collisions and internal hadron struc- ture, Nucl. Phys. B 86, 243 (1975), http://dx.doi. org/10.1016/0550-3213(75)90443-5

[25] R.C. Hwa, Evidence for valence-quark clusters in nucleon structure functions, Phys. Rev. D 22, 759 (1980), http://dx.doi.org/10.1103/PhysRevD.22.759

PROTONŲ SANDAROS PASIREIŠKIMAS KETEROS FORMOS KORELIACIJOMIS, SUSIDURIANT AUKŠTOS ENERGIJOS PROTONAMS

P. Kubiczek ir S.D. Głazek

${ }^{\text {a } J o g a i l o s ~ u n i v e r s i t e t a s, ~ K r o k u v a, ~ L e n k i j a ~}$

${ }^{\mathrm{b}}$ Varšuvos universitetas, Varšuva, Lenkija 